\title{
Procura da emergência pediátrica pelas mães: implicações para a superlotação
}

Mothers searching for pediatric emergency: implication on overcrowding at the emergency units

Libania Maria Bandeira Lima', Nádia Maria Girão Saraiva de Almeida²

${ }^{1}$ Mestre em Saúde da Criança e do Adolescente pela Universidade Estadual do Ceará (UECE) - Fortaleza (CE), Brasil. Médica de Família e Comunidade e Pediatra, junto à Secretaria Municipal de Saúde de Fortaleza (CE), Brasil.

marlijc2207@hotmail.com

2 Doutora em Saúde Pública pela Universidade de São Paulo - São (SP), Brasil. Professora Colaboradora do Curso de

Mestrado Profissional em Saúde da Criança e

do Adolescente da Universidade Estadual do

Ceará (UECE) - Fortaleza (CE), Brasil.

nadiagirao@gmail.com
RESUMO $O$ estudo objetivou traçar o perfil das crianças e adolescentes que buscam o atendimento no Centro de Assistência à Criança Dra. Lúcia de Fátima Rodrigues Guimarães Sá (CAC), em Fortaleza/Ceará, descrever os vínculos com os serviços de atenção básica e as justificativas das mães pela procura do serviço de emergência. Estudo descritivo, transversal, realizado no período de $1^{\circ}$ a 31 de maio de 2011, cuja amostra foi constituída por 211 participantes. Uma parcela de $47,4 \%$ dos atendimentos foi classificada como patologias que poderiam ser conduzidas na atenção básica. A procura das mães pela emergência pediátrica foi justificada pela qualidade do atendimento, pela acessibilidade e, em algumas ocasiões, pela ausência do médico na Unidade Básica de Saúde.

PALAVRAS CHAVE: Superlotação de emergências pediátricas; atenção básica; hierarquização na saúde.

ABSTRACT The study aimed to outline the profile of children and adolescents who seek care at the Child Care Center Dr. Lucia de Fatima Rodrigues Guimarães Sá (Centro de Assistência à Criança-CAC), in Fortaleza / Ceará, describe the links with primary care services and the justifcations of mothers looking for emergency service. Descriptive transversal study, conducted from 1 to 31 May 2011, whose sample consisted of 211 participants. A portion of $47.4 \%$ of visits were classified as pathologies that could be conducted in primary care. The search of mothers for pediatric emergency was justified by the quality of care, the accessibility and, in some instances, the lack of doctors in the Basic Health Units.

KEYWORDS: Overcrowding of pediatric emergencies; primary care; prioritization in health. 


\section{Introdução}

No Brasil, com a Constituição de 1988, foram lançadas as bases da implementação do Sistema Único de Saúde (SUS), que mudou a história da saúde pública no país. O SUS está fundamentado sobre os princípios norteadores orientados pela conferência de Alma-Ata e descritos pela Lei Orgânica da Saúde, Lei 8080/90, sendo estes éticos/doutrinários: Integralidade, Equidade e Universalidade; e organizativos/operacionais: descentralização, participação da comunidade, hierarquização e regionalização (BRASIL, 2000).

Entende-se como Atenção Primária à Saúde (APS) a porta de entrada do Sistema Único de Saúde, englobando as Unidades Básicas de Saúde com a Estratégia Saúde da Família (ESF), estratégia essa que se caracteriza por utilizar-se de equipes multiprofissionais responsáveis por oferecer atenção integral e contínua a indivíduos e suas famílias residentes em um território rural ou urbano, com limites geográficos definidos, com resolubilidade e qualidade da atenção (BRASIL, 1998).

A cidade de Fortaleza, capital do estado do Ceará, é uma das maiores metrópoles do Brasil, sendo a $5^{\text {a }}$ maior em número de habitantes, com uma população de 2.447.409 pessoas (INSTITUTO BRASILEIRO DE GEOGRAFIA E ESTATÍSTICA, 2010).

Estudiosos concordam que Fortaleza é um local de contrastes sociais, onde se destaca a convivência de modernos aparatos industriais, financeiros, turísticos e luxuosas áreas residenciais com uma população pobre e excluída, usuária da assistência social e carente de políticas públicas comprometidas com o desenvolvimento humano sustentável (LIMA, 2004).

O Centro de Assistência à Criança Lúcia de Fátima Rodrigues Guimarães Sá (CAC), único hospital pediátrico da Prefeitura Municipal de Fortaleza, realiza em média 8.000 atendimentos mensais, entre ambulatórios, urgência/emergência e internamentos. Em cada plantão de 12 horas devem atuar três pediatras, que se revezam entre os setores de emergência, enfermaria e pronto atendimento (FORTALEZA, 2010).

Sobre a recepção das crianças e adolescentes que chegam ao hospital, uma equipe de enfermagem, realiza a triagem (acolhimento com classificação de risco), baseada nas queixas e condição clínica, utilizando protocolo adaptado para o serviço. Aqueles pacientes que forem classificados como pertencentes ao eixo vermelho (emergência) são encaminhados imediatamente para o setor de emergência, onde receberão atendimento médico imediato. Os demais são submetidos à aferição de sinais vitais e encaminhados ao Serviço de Arquivo Médico e Estatística (SAME) para a confecção da ficha de atendimento. Posteriormente, serão atendidos segundo a classificação de risco (amarelo ou verde) (FORTALEZA, 2010).

Os pacientes classificados como pertencentes ao eixo azul são orientados a procurar uma Unidade Básica de Saúde, local hierarquicamente adequado para o seu cuidado. Na prática, entretanto, não se pode negar assistência médica a quem necessita, especialmente pelo respeito aos princípios do SUS. Como as mães relutam em seguir a orientação dada, acabam por ficar no espaço de espera, na esperança de receber atendimento, atitude que contribui para a superlotação no serviço. Em períodos de maior demanda esses pacientes chegam a aguardar um dia inteiro e ainda assim retornam às suas casas sem receber a assistência.

O quadro descrito gera, para os familiares e cuidadores dos pacientes e para os profissionais de saúde, relaçóes delicadas e conflituosas, chegando muitas vezes a cenas de agressão verbal e até violência física, o que configura esses ambientes de trabalho como zonas de insegurança e fragilidade social, fatores de desestímulo para os trabalhadores da saúde e de desesperança e revolta para os usuários do sistema.

Este trabalho se propõe a avaliar essa desconfortável condição de risco à qual estão expostas tantas crianças e adolescentes de Fortaleza e, a partir de uma melhor compreensão, contribuir para estratégias de aperfeiçoamento da qualidade dos serviços prestados tanto para os usuários como para os trabalhadores da saúde desse município, bem como colaborar para políticas públicas que trabalhem as razóes das mães, no sentido de orientar uma busca adequada pelo serviço cuja necessidade venha a ocorrer.

O estudo teve por objetivo traçar o perfil das crianças e adolescentes que buscam o atendimento no Centro de Assistência à Criança Dra. Lúcia de Fátima 
Rodrigues Guimarães Sá (CAC), em Fortaleza/Ceará, descrever os vínculos com os serviços de atenção básica e as justificativas das mães pela procura do serviço de emergência.

\section{Metodologia}

Estudo descritivo, transversal, realizado no setor de emergência e pronto atendimento do CAC, em Fortaleza, no período de $1^{\circ}$ a 31 de maio de 2011, excluídos os feriados e fins de semana, quando os postos de saúde não funcionam.

A população do estudo foi constituída por crianças/adolescentes e suas mães/acompanhantes que buscaram atendimento de urgência/emergência no CAC no período definido e que preenchiam os seguintes critérios de inclusão: residir no município de Fortaleza/ Ceará; ser classificado como pertencente ao eixo azul segundo o protocolo de acolhimento com classificação de risco do CAC estando, portanto, apto para o atendimento na atenção básica; buscar o atendimento hospitalar em horário coincidente com o funcionamento da maioria dos postos de saúde da Rede Básica de Fortaleza: de segunda a sexta-feira, das 7:00h às 17:00h. Foram excluídos os pacientes com diagnóstico confirmado ou suspeito de dengue, por estar justificado buscar atendimento médico em um período de epidemia.

Uma análise das fichas dos atendimentos realizados nos meses de março e setembro de 2010 mostrou que 1.200 pacientes tiveram o diagnóstico de agravos que poderiam ser resolvidos na atenção básica, correspondendo a uma prevalência de $45 \%$. Assim, com base nesses resultados, calculou-se o tamanho da amostra utilizando-se a fórmula para populaçóes finitas:

$$
\mathrm{n}=\frac{\mathrm{t}^{2} 5 \% x \mathrm{PxQ} \mathrm{xN}}{\mathrm{e}^{2}(\mathrm{~N}-1)+\mathrm{t}^{2} 5 \% \mathrm{xPxQxN}}
$$

onde $\mathrm{N}$ corresponde à população de 1.200 crianças atendidas mensalmente no CAC com diagnósticos passíveis de atendimento na atenção básica; P é a prevalência de $45 \%$ desses agravos. Empregou-se o erro amostral (e) de 6\%. Dessa forma, a amostra ficou constituída por 216 pacientes.
Considerando o período de 20 dias úteis para a coleta de dados, definiu-se que diariamente seriam selecionados 11 pacientes para participar da pesquisa, sendo realizado o sorteio, empregando-se a tabela de números aleatórios.

As informaçóes foram obtidas por meio de entrevistas com as mães, utilizando um formulário contendo variáveis sociodemográficas, vinculaçáo aos serviços de atenção básica, dados clínicos da doença atual e os motivos da busca pelo hospital. Constava, ainda, no formulário um espaço a ser preenchido com informaçóes colhidas após a consulta médica, como tempo de espera, diagnóstico e tratamento instituído, que foram coletados dos prontuários, posteriormente.

Dois profissionais previamente treinados pela pesquisadora realizaram o sorteio e as entrevistas. Após a leitura do Termo de Consentimento Livre e Esclarecido, as mães/acompanhantes que concordaram em participar do estudo assinaram o termo, em duas vias, guardando uma para si e deixando outra com o entrevistador. A entrevista foi realizada no momento em que a criança aguardava $o$ atendimento.

Durante a revisão do instrumento de coleta de dados, a pesquisadora responsável identificou que os formulários de duas crianças e três adolescentes apresentavam o preenchimento incompleto de diversas variáveis, sendo excluídos do estudo, ficando a amostra final constituída por 211 participantes.

Os dados foram tabulados no programa SPSS, versão 16.0 e apresentados sob a forma de tabelas. Foram calculadas médias e proporçôes e realizada análise estatística utilizando-se o teste qui-quadrado para verificar a associação entre as variáveis.

O projeto foi aprovado pelo Comitê de Ética em Pesquisa da Universidade Estadual do Ceará. A pesquisa se pautou nos princípios da Resolução 196/96 do Conselho Nacional de Saúde/Ministério da Saúde (CONSELHO NACIONAL DE SAÚDE, 1996).

\section{Resultados}

No mês de maio de 2011, excluídos feriados e finais de semana, foram realizados 2.885 atendimentos, dos quais $14,4 \%$ (416) não receberam diagnóstico. Entre os que 
foram diagnosticados (2.469), uma parcela de $47,4 \%$ (1.170) foi classificada como patologias que poderiam ser conduzidas na atenção básica, sendo considerados os pacientes que sofriam de sintomas leves, onde não havia qualquer possibilidade real de complicação. Predominaram as doenças do trato respiratório (56,2\%), seguidas das doenças gastrointestinais $(16,6 \%)$ e das viroses $(13,1 \%)$.
A maioria das crianças encontrava-se na faixa etária de zero a 24 meses $(37,0 \%)$, enquanto os adolescentes ( $\geq 10$ anos) representaram 13,7\% da amostra, destacando-se também o predomínio do sexo masculino $(53,1 \%)$. Com relação ao vínculo dos acompanhantes, $81,5 \%$ foram trazidos pelas mães, $6,2 \%$ pelas avós e apenas $5,7 \%$ estavam acompanhadas pelos pais (Tabela 1).

Tabela 1. Distribuição das crianças segundo idade, sexo e vínculo do acompanhante. Fortaleza, 2011

\begin{tabular}{llc}
\hline Idade (meses) $(\mathbf{n}=\mathbf{2 1 1})$ & $\mathbf{n .}$ & $\%$ \\
\hline$<1$ & 5 & 2,4 \\
\hline $1-11$ & 22 & 10,4 \\
\hline $12-24$ & 51 & 24,2 \\
\hline $25-59$ & 49 & 23,2 \\
\hline $60-120$ & 55 & 26,1 \\
\hline$>120$ & 29 & 13,7 \\
\hline
\end{tabular}

$\operatorname{Sexo}(n=211)$

\begin{tabular}{lccc}
\hline Masculino & 112 & 53,1 \\
\hline Feminino & 99 & 46,9 & \\
\hline
\end{tabular}

Vínculo com a criança $(\mathbf{n}=\mathbf{2 1 1})$

\begin{tabular}{lcc}
\hline Mãe & 172 & 81,5 \\
\hline Pai & 12 & 5,7 \\
\hline Avô (ó) & 13 & 6,2 \\
\hline Tio (a) & 11 & 5,2 \\
\hline Outros & 3 & 1,4 \\
\hline
\end{tabular}

Fonte: Elaboração própria

Quanto ao perfil dos acompanhantes, a idade variou de 15 a 63 anos, sendo a maioria $(42,2 \%)$ na faixa etária de 20 a 29 anos, predominando os que não completaram o ensino fundamental $(33,5 \%)$. No quesito renda familiar, $84,4 \%$ referiram renda mensal de um a dois salários mínimos enquanto que 11,2\% recebiam menos que um salário mensal.
Sobre a procedência dos pacientes, verificouse que $91,0 \%$ vieram diretamente de seus domicílios para o serviço de emergência, enquanto apenas 7,1\% foram referenciados pela Unidade Básica de Saúde. Após a classificação de risco, 57,3\% (121) foram encaminhados para a consulta de pronto atendimento. Os demais foram orientados a procurar assistência em 
outros serviços. Entre os que aguardaram pelo atendimento, apenas $14,8 \%(18 / 121)$ obtiveram a consulta médica dentro da primeira hora, enquanto $17,4 \%$ $(21 / 121)$ permaneceram mais de seis horas à espera da avaliaçấo pediátrica, sendo a média de tempo de espera de 2 horas e 15 minutos. Ressalta-se que em 34,6\% dos atendimentos analisados (73/211) havia apenas um médico no turno, enquanto que 42,2\% (89/211) contaram com dois médicos e, em 23,2\% dos casos, três ou quatro médicos realizaram o atendimento.
A maioria dos pacientes encontrava-se cadastrada em uma Unidade Básica de Saúde (93,4\%), desses 67,5\% na unidade do próprio bairro. Quanto ao acompanhamento do desenvolvimento normal da criança e do adolescente, 55,0\% responderam que não o fazem, dos quais 39,6\% justificaram-se pela dificuldade de acesso e $38,8 \%$ disseram desconhecer ou não gostar dos serviços oferecidos pela atenção primária. Dos que faziam seguimento regular no posto de saúde, $32,6 \%$ referiram ter conhecimento da necessidade desse cuidado (Tabela 2).

Tabela 2. Vinculação dos pacientes à atenção básica. Fortaleza, 2011

\begin{tabular}{|c|c|c|}
\hline Cadastrado em uma Unidade Básica de Saúde ( $(n=211)$ & n. & $\%$ \\
\hline Sim & 197 & 93,4 \\
\hline Não & 10 & 4,7 \\
\hline Não respondeu/não sabe informar & 4 & 1,9 \\
\hline \multicolumn{3}{|l|}{ Unidade Básica de Saúde em que é cadastrado (n=197) } \\
\hline Unidade Básica de Saúde do bairro onde reside & 133 & 67,5 \\
\hline Unidade Básica de Saúde de outro bairro & 64 & 32,5 \\
\hline \multicolumn{3}{|l|}{ Faz acompanhamento na Unidade Básica de Saúde $(\mathbf{n = 2 1 1 )}$} \\
\hline Não & 116 & 55,0 \\
\hline Sim & 92 & 43,6 \\
\hline Não respondeu & 3 & 1,4 \\
\hline \multicolumn{3}{|l|}{$\begin{array}{l}\text { Justificativas para não fazer acompanhamento na Unidade } \\
\text { Básica de Saúde }(n=116)\end{array}$} \\
\hline Dificuldade de acesso & 46 & 39,6 \\
\hline Desconhece/não gosta do serviço & 45 & 38,8 \\
\hline Faz seguimento em outro serviço & 3 & 2,6 \\
\hline Planeja fazer & 3 & 2,6 \\
\hline Acompanhante não respondeu & 19 & 16,4 \\
\hline \multicolumn{3}{|c|}{$\begin{array}{l}\text { Justificativas para fazer acompanhamento na Unidade Básica } \\
\text { de Saúde }(n=92)\end{array}$} \\
\hline Acompanhante não respondeu & 31 & 33,7 \\
\hline Responsável entende a necessidade & 30 & 32,6 \\
\hline Por orientação do próprio serviço & 19 & 20,7 \\
\hline Paciente tem doença que exige o acompanhamento & 12 & 13,0 \\
\hline
\end{tabular}

Fonte: Elaboração própria 
Verificou-se que elevada proporção dos entrevistados preferia procurar o CAC $(78,2 \%)$, enquanto apenas $10,0 \%$ buscavam a Unidade Básica quando a criança/adolescente adoecia. Dentre os que preferiam o CAC, $26,1 \%$ afirmaram gostar do atendimento, $21,2 \%$ justificaram a escolha pela acessibilidade e 9,7\% optam pelo hospital por insatisfação e/ou pela dificuldade de acesso à Unidade Básica de Saúde. As razóes predominantes para procurar a Unidade Básica de saúde foram a acessibilidade/proximidade $(28,6 \%)$ e gostar do atendimento $(23,8 \%)$ (Tabela 3$)$.

Tabela 3. Serviços procurados e razão da preferência em casos de doença da criança/adolescente. Fortaleza, 2011

\begin{tabular}{lcc}
\hline Serviços procurados em casos de doença $(\mathbf{n = 2 1 1 )}$ & $\mathbf{n .}$ & $\%$ \\
\hline CAC & 165 & 78,2 \\
\hline Unidade Básica de Saúde & 21 & 10,0 \\
\hline Outro hospital público & 13 & 6,1 \\
\hline Serviço particular & $\mathbf{5}$ & $\mathbf{2 , 4}$ \\
\hline Farmácia & 1 & 0,5 \\
\hline Não responderam & 6 & 2,8 \\
\hline
\end{tabular}

\section{Razões da preferência pelo CAC ( $n=165)$}

\begin{tabular}{lcc}
\hline Gostam do atendimento & 43 & 26,1 \\
\hline Acessibilidade & 35 & 21,2 \\
\hline Valorizam serviço especializado/exames & $\mathbf{2 2}$ & $\mathbf{1 3 , 3}$ \\
\hline Dificuldade de acesso/insatisfação com a unidade básica & $\mathbf{1 6}$ & $\mathbf{9 , 7}$ \\
\hline Seguem critérios de gravidade da doença & 6 & 3,6 \\
\hline Não responderam & 43 & 26,1 \\
\hline
\end{tabular}

\section{Razões da preferência pela Unidade Básica de Saúde $(n=21)$}

\begin{tabular}{llc}
\hline Acessibilidade/proximidade & 6 & 28,6 \\
\hline Gostam do atendimento & 5 & 23,8 \\
\hline Seguem critérios de gravidade da doença & $\mathbf{3}$ & $\mathbf{1 4 , 3}$ \\
\hline Não responderam & 7 & 33,3 \\
\hline
\end{tabular}

Fonte: Elaboração própria

Observou-se que $86,7 \%$ dos entrevistados consideraram a doença da criança/adolescente como emergência. Entre esses, a maioria justificou ser caso de emergência por temer complicaçóes $(41,0 \%)$, porque a criança apresentava febre $(22,4 \%)$ e $20,2 \%$ achavam que poderia ser dengue.
Quando interrogados sobre a procura por outros serviços na doença atual da criança/adolescente, 64,9\% dos entrevistados responderam que vieram diretamente ao hospital. Dos 73 que buscaram outro serviço antes de ir ao CAC, a maioria (76,7\%) afirmou ter ido à Unidade Básica de Saúde. Quanto aos motivos pela escolha 
do CAC no dia da entrevista, 37,0\% destacaram proximidade e acessibilidade, $21,3 \%$ afirmaram que gostam do atendimento no hospital, ressaltando-se que 9,0\% afirmaram ter vindo à emergência porque não havia médico nas Unidades Básicas de Saúde (Tabela 4).

\begin{tabular}{lcc} 
Tabela 4. Serviços procurados e razão da preferência em casos de doença da criança/adolescente. Fortaleza, 2011 & $\%$ \\
\hline Procura por outro serviço antes de vir ao CAC (n=211) & $\mathbf{n}$. & 34,6 \\
\hline Sim & 73 & 64,9 \\
\hline Não & 137 & 0,5 \\
\hline Não respondeu & 1 & $\%$ \\
\hline
\end{tabular}

\section{Serviços que foram procurados $(n=73)$}

\begin{tabular}{lcc}
\hline Unidade Básica de Saúde & 56 & 76,7 \\
\hline Dois ou mais serviços & 8 & 11,0 \\
\hline Outro hospital & 7 & 9,6 \\
\hline Farmácia & 2 & 2,7 \\
\hline
\end{tabular}

\section{Motivos para procurar o CAC na doença atual $(n=206)$}

\begin{tabular}{lcc}
\hline Gostam do atendimento & 45 & 21,8 \\
\hline Proximidade & 42 & 20,4 \\
\hline Acessibilidade & 36 & 17,5 \\
\hline Hospital de Emergência Pediátrica & 21 & 10,2 \\
\hline Não tem médico na Unidade Básica de Saúde & 19 & 9,2 \\
\hline Por encaminhamento/sugestão & 16 & 7,8 \\
\hline Por costume/hábito & 10 & 4,8 \\
\hline Por medo de complicação & 9 & 4,4 \\
\hline Por ter acesso a exames & 8 & 3,9 \\
\hline
\end{tabular}

Fonte: Elaboração própria

Acerca das expectativas das mães/acompanhantes em relação ao atendimento de suas crianças, a maior parte $(35,5 \%)$ espera agilidade, $16,6 \%$ qualidade da atenção e 13,3\% almejam simplesmente ser atendidas (13,3\%). No quesito sugestóes para melhorar o atendimento no município, 102 entrevistados não quiseram opinar e entre os que o fizeram predominou a sugestão de aumentar o número de médicos na rede municipal, perfazendo $21,8 \%$. Outros 15,2\% lembraram a necessidade de melhorar a organização dos serviços de emergência (Tabela 5) 
Tabela 5. Expectativas e sugestões dos entrevistados em relação aos serviços de saúde. Fortaleza, 2011

\begin{tabular}{|c|c|c|}
\hline $\begin{array}{l}\text { Expectativas em relação ao atendimento na emergência } \\
(n=210)\end{array}$ & n. & $\%$ \\
\hline Agilidade & 75 & 35,5 \\
\hline Qualidade & 35 & 16,6 \\
\hline Ser atendido(a) & 28 & 13,3 \\
\hline Melhor organização do Serviço & 21 & 10,0 \\
\hline Resolutividade & 19 & 9,0 \\
\hline Agilidade/Qualidade/Resolutividade & 13 & 6,1 \\
\hline Não responderam & 19 & 9,0 \\
\hline \multicolumn{3}{|l|}{$\begin{array}{l}\text { Sugestões para melhorar o atendimento no município } \\
(n=109)\end{array}$} \\
\hline Aumentar o número de médicos & 46 & 21,8 \\
\hline Melhorar a organização do Serviço de Emergência & 32 & 15,2 \\
\hline Estão satisfeitos(as) & 18 & 8,5 \\
\hline Melhorar a qualidade do atendimento médico & 7 & 3,3 \\
\hline Ampliar a rede de atendimentos e funcionários & 6 & 2,9 \\
\hline
\end{tabular}

Fonte: Elaboração própria

\section{Discussão}

No hospital da rede secundária de Fortaleza, local destinado ao atendimento de urgência e emergência, praticamente metade da demanda correspondeu a patologias que poderiam ter sido resolvidas na Atenção Básica em Saúde, resultado semelhante a outros estudos sobre perfil de morbidade da clientela de prontos-socorros.

Melo, Assunção e Ferreira (2007) descrevem como paradoxal o contexto de um serviço de emergência pediátrica na cidade de Belo Horizonte/MG que se apresenta constantemente lotado, aglomerado de crianças e de seus acompanhantes, entretanto $50 \%$ dos casos foram considerados como consultas simples, sensíveis a atendimento em serviços de cuidados primários.

Rati (2009) explica esse paradoxo mostrando as justificativas das máes para optar por serviços de emergência em uma cidade como Belo Horizonte. Apesar de ter em 2008 uma cobertura de 75\% de sua população pelo Programa Saúde da Família e contar com mais 14 portas de entrada de urgência no município, sendo sete de Unidades de Pronto Atendimento e sete de Pronto-Socorro Hospitalar, além de sistemas de triagem e classificação de risco estruturados com o empenho de esclarecer a população sobre critérios de gravidade, ainda não se verificara a redução do número de atendimentos ambulatoriais nas emergências hospitalares.

Entre os pacientes atendidos no CAC no período em estudo e classificados como isentos de risco iminente, verificou-se que a maioria estava acometida por agravos do trato respiratório seguidos por transtornos de natureza gastrointestinal. Oliveira e Scochi (2002), e Riccetto et al. (2007) também encontraram 
predomínio das patologias respiratórias em serviços de emergência clínica.

A procura do serviço pelo público adolescente é bem reduzida quando comparada com as demais faixas etárias, evidenciando-se que a maior parte da demanda se encontra nos dois primeiros anos de vida, seguida pelos escolares de 5 a 10 anos. Riccetto et al. (2007) e Veras et al. (2009) também encontraram predominância de pacientes menores de cinco anos em pesquisas realizadas em emergências pediátricas.

Para Ferrari, Thomson e Melchior (2006), é difícil afirmar se a notória ausência dos adolescentes nos serviços de saúde se deve à baixa procura ou à pouca oferta de açóes voltadas para eles. $\mathrm{O}$ estudo revela que mesmo dentro da Estratégia Saúde da Família as açóes direcionadas aos adolescentes ocorrem de forma isolada e desarticulada, não representando um trabalho intersetorial expressivo e não estabelecido como prioridade em meio a outros programas com maior demanda.

O predomínio do sexo masculino também foi encontrado nos estudos de Riccetto et al. (2007) e Veras et al. (2009), conduzidos em serviços de emergência pediátrica.

Acerca da procedência, chama a atenção que a maioria dos pacientes tenha vindo diretamente de seus domicílios para o serviço de emergência, o que pode ser explicado pelo elevado percentual de crianças que não são acompanhadas na Unidade Básica de Saúde, por dificuldade de acesso ou porque suas mães não gostam do serviço ofertado.

Resultados semelhantes foram obtidos por Batistela, Guerreiro, Rosseto (2008), em pesquisa realizada em pronto-socorro pediátrico na cidade de Londrina quando verificaram a procura direta em $56,76 \%$ dos entrevistados.

$\mathrm{Na}$ opinião de Melo, Assunção e Ferreira (2007), os médicos justificam a necessidade de atender essas crianças porque seria arriscado devolvê-las ao posto de saúde sem a certeza de assistência. Algumas dessas crianças poderiam ter seu quadro agravado nos dias subsequentes, especialmente aquelas de menor idade.

Stein (1998) demonstra que a população precisa ser acolhida, embora em uma melhor organização devesse sair com um encaminhamento para o seu local de direito, o atendimento médico continuado.

Não é suficiente orientar a procurar assistência em outros serviços, uma vez que a população está acostumada a ouvir e divulgar entre si que faltam médicos nos postos de saúde e que o atendimento no hospital, embora demorado, é mais acessível. Entre os que aguardaram pelo atendimento no CAC, o tempo de espera variou entre menos de uma hora e mais de seis horas à espera da avaliação pediátrica.

Melo, Assunção e Ferreira (2007) encontraram um tempo de espera pelo atendimento em emergência pediátrica de Belo Horizonte que se aproxima dos resultados de Fortaleza descritos neste estudo. Segundo esses pesquisadores, nos períodos de maior demanda um paciente pode esperar até sete horas, gerando um quadro de angústia, desconforto e irritabilidade nos usuários.

A Estratégia Saúde da Família no município de Fortaleza ainda se encontra em fase de implantaçáo, tendo alcançado discreto progresso nos últimos anos. Apesar de uma elevada proporção de pacientes estar cadastrada em um posto de saúde, muitos são cadastrados em postos de outro bairro, o que gera dificuldade de acesso e faz com que o acompanhamento do desenvolvimento normal da criança não seja viável. Entre aqueles que fazem seguimento regular nos postos, cerca de um terço afirmou ter conhecimento da importância do acompanhamento.

Starfield (1994), em um estudo ecológico em 1994, realizou uma comparação entre 11 países para identificar as relaçóes entre o grau de orientação à APS dos sistemas de saúde e os indicadores de saúde nacionais. A conclusão de seu trabalho foi que

\section{[...] quanto maior o grau de orientação do siste- ma de saúde de cada país à APS, maior o grau de satisfação de sua população com o sistema de saúde, melhores os indicadores de saúde (baixo peso ao nascer, mortalidade infantil, expectati- va de vida, anos potenciais de vida perdidos), menores os custos totais com o cuidado em saú- de e menor o consumo individual de medica- mentos. (HARZHEIM; STEIN; DARDET; 2004 apud STARFIELD, 1994, p. 31).}


Verificou-se que a maioria dos acompanhantes das crianças considera ser o seu caso de emergência, porém suas justificativas não se baseiam em critérios concretos, clinicamente detectáveis, mas sim em sentimentos inquietantes para si como o medo de complicações ou de ser doença grave. Entre os sintomas assustadores para os pais, a febre vem em primeiro lugar, seguida por dor, tosse e vômitos.

Resultados semelhantes são descritos por Batistela, Guerreiro e Rosseto (2008), que afirmam haver total desconhecimento pela populaçấo sobre a função dos diferentes níveis de complexidade dos serviços de saúde e sobre a organizaçáo dos mesmos, gerando mais entraves e desafios.

Dentre os motivos citados pelos entrevistados para procurarem o CAC no dia da entrevista, destacam-se a proximidade e a acessibilidade como principais razóes pela escolha, merecendo destaque a falta de médicos na Unidade Básica de Saúde.

Segundo Kovacs et al. (2005), em um estudo sobre a acessibilidade de crianças aos serviços de atenção básica em Recife, existe descompasso entre a necessidade de atenção e o crescimento da rede básica, e indefinições acerca do seu papel na percepção dos usuários, que não compreendem a menor oferta de consultas médicas e ainda não se habituaram com as consultas de enfermagem. O usuário também não apresenta boa definição entre consulta eletiva e casos de urgência/emergência, baseando-se em seus próprios critérios na hora de procurar os serviços.

Quanto às sugestóes, a maior parte dos entrevistados não quis opinar e acrescentou não acreditar em possibilidade de melhorias, demonstrando assim uma notável sensação de descrédito para com as açóes da administração pública. Dentre os que opinaram para a melhoria do atendimento, citam-se aumentar o número de médicos na rede municipal e melhorar a organização dos serviços de emergência.

\section{Conclusões}

As crianças/adolescentes atendidas na emergência eram, na maioria, do sexo masculino, filhos de mães com baixa escolaridade e renda familiar até dois salários mínimos.

Embora uma elevada proporção fosse cadastrada em uma Unidade Básica de Saúde, um considerável percentual não fazia acompanhamento regular nesses serviços, sendo as principais justificativas a dificuldade de acesso e o descontentamento com o serviço ofertado.

A procura das máes pelo serviço de emergência pediátrica, mesmo quando as crianças apresentavam queixas não urgentes, foi justificada pela qualidade do atendimento, pela acessibilidade e, em algumas ocasióes, pela ausência do médico na Unidade Básica de Saúde.

A elevada proporção de crianças com problemas de saúde passíveis de serem solucionados na atenção básica repercute sobre a qualidade da assistência prestada não só àqueles que realmente necessitam dos serviços de urgência, mas também aos que precisam de atenção ambulatorial, pois o atendimento objetiva resolver a queixa do paciente e este, muitas vezes, não é encaminhado para a Unidade Básica, comprometendo, assim, a integralidade da atenção.

\section{Referências}

BATISTELA, S.; GUERREIRO, N. P.; ROSSETTO, E. G. Os motivos de procura pelo Pronto-Socorro Pediátrico de um Hospital Universitário referidos pelos pais ou responsáveis. Semina: Ciências Biológicas e da Saúde, Londrina, v. 29, n. 2, p. 121-130, jul./dez. 2008. Disponível em: <http://www.uel.br/revistas/uel/ index.php/seminabio/article/viewFile/3460/2 815>. Acesso em: 21 jan. 2011
BRASIL. Ministério da Saúde. Manual para a Organização da Atenção Básica. Brasília: Ministério da Saúde, 1998. 40 p. Disponível em: <http://dab.saude.gov.br/docs/geral/manual _organizacao_ab.pdf>. Acesso em: 17 fev. 2012.

Ministério da Saúde. Secretaria Executiva. Sistema

Único de Saúde (SUS): princípios e conquistas. Brasília: Ministério da Saúde, 2000. 44 p. Disponível em: <http:// 
bvsms.saude.gov.br/bvs/publicacoes/sus_principios.pdf>. Acesso em: 19 jan. 2011.

CONSELHO NACIONAL DE SAÚDE. Resolução 196/96, decreto n. 93.993, de janeiro de 1987. Estabelece critérios sobre pesquisa envolvendo seres humanos. Bioética, Brasília, v. 4, n. 2, supl., p. 15$22,1996$.

FERRARI, R. A. P; THOMSON, Z:; MELCHIOR, R. Atenção à saúde dos adolescentes: percepção dos médicos e enfermeiros das equipes da saúde da família. Cadernos de Saúde Pública, Rio de Janeiro, v. 22, n. 11, p. 2491-2495, nov. 2006. Disponível em: <www.scielo.br/ pdf/csp/v22n11/24.pdf>. Acesso em: 09 mar. 2012.

FORTALEZA. Secretaria Municipal de Saúde. Rede Hospitalar e Atenção Básica: dados de 2010. Disponível em: <http://www. sms.fortaleza.ce.gov.br/sms_v2/redes_redeHospitalar_CAC.asp>. Acesso em: 21 jan. 2011.

HARZHEIM, E.; STEIN, A.T.; DARDET, C. A. A efetividade dos atributos da Atenção Primária sobre a saúde infantil. Boletim da Saúde, Porto Alegre, v. 18, n. 1, jan/jun. 2004. Disponível em: <http://www.esp. rs.gov.br/img2/v18n1_05\%20efetividadeatrib.pdf>. Acesso em: 29 mar. 2012.

INSTITUTO BRASILEIRO DE GEOGRAFIA E ESTATÍSTICA. Censo demográfico 2010. Disponível em: <www.censo2010.ibge.gov. br/>. Acesso em: 26 fev. 2011.

KOVACS, M. H. et al. Acessibilidade às ações básicas entre crianças atendidas em serviços de pronto-socorro. Jornal de Pediatria, Rio de Janeiro, v. 81, n. 3, p. 251-258, jun. 2005. Disponível em: <http://www.scielo.br/scielo.php?script=sci_ arttext\&pid=\$0021-75572005000 400013 $>$. Acesso em: 03 set. 2010.

LIMA, J. L.C. A cidade de Fortaleza: crise urbanae desigualdade social. Emancipação, Ponta Grossa, v. 4, n. 1, pp. 73-84, 2004. Disponível em: <www.revistas2.uepg.br/index.php /emancipacao/article / view/49/46.>. Acesso em: 25 fev. 2011.

MELO, E. M. C.; ASSUNÇÃO, A. A.; FERREIRA, R. A. O trabalho dos pediatras em um serviço público de urgências: fatores intervenientes no atendimento. Cadernos de Saúde Pública, Rio de Janeiro, v. 23, n. 12, p. 3000-3010, dez. 2007. Disponível em: <http://www.scielo.br/pdf/csp/v23n12/20.pdf>. Acesso em: 10 fev. 2012.

OLIVEIRA, M. L. F.; SCOCHI, M. J. Determinantes da utilização dos serviços de urgência/ emergência em Maringá (PR). Revista Ciência, Cuidado e Saúde, Maringá, v. 1, n. 1, p. 123128, 1. sem. 2002. Disponível em:<http://periodicos.uem. br/ojs/index.php/CiencCuidSaude>. Acesso em: 03 set. 2010.

RATI, R. M. S. "Criança não pode esperar": a demanda de mães e suas crianças em condições não urgentes em ambulatório pediátrico de urgência e emergência. 2009. 121 f. Dissertação (Mestrado em Ciências da Saúde) Universidade Federal de Minas Gerais, Belo Horizonte, 2009. Disponível em: <http://www.medicina.ufmg.br/ cpg/programas/saude_crianca/teses_dissert/2009_ mestrado_RoseMeireRati.pdf>. Acesso em: 21 jan. 2010.

RICCETTO, A. G. L. et. al. Sala de emergência em pediatria: casuística de um hospital universitário. Revista Paulista de Pediatria, São Paulo, v. 25, n. 2, p. 156-160, jun. 2007. Disponível em: <http://www.scielo.br/scielo. php?pid=S0103-05822007000200010\&script $=\quad$ sci_ arttext>. Acesso em: 16 mar. 2012.

STEIN, T. A. Acesso a atendimento médico continuado: uma estratégia para reduzir a utilização de consultas não urgentes em serviços de emergência. 1998. 248 f. Tese (Doutorado) - Faculdade de Medicina da Universidade Federal do Rio Grande do Sul. Curso de Pósgraduação em Medicina: Clínica médica. Porto Alegre. Disponível em: <http://www.lume.ufrgs.br/bitstream/ handle/10183/1919/000223165. pdf? sequence $=1>$. Acesso em: 10 fev. 2012.

VERAS, J. E. G. L. F. et al. Análise das causas de atendimento de crianças e adolescentes menores de 15 anos em Pronto Atendimento de um Hospital Secundário de Fortaleza. In: CONGRESSO BRASILEIRO DE ENFERMAGEM, 61, 2009, FORTALEZA. Anais... Fortaleza: Congresso, 2009. Disponível em: <http://www.abeneventos.com.br/ anais_61cben /files/00961.pdf>. Acesso em: 10 fev. 2012.

Recebido para publicação em Outubro/2011

Versão final em Junho/2012

Conflito de interesse: Não houve.

suporte financeiro: Inexistente. 